
\title{
$\angle$ Research Square \\ Pancreaticline Cell Differentiation of Bone Marrow Mesenchymal Stromal Cells in Acellular Pancreatic Scaffolds
}

\section{Zhao Li}

Peking University People's Hospital

\section{Yue Du}

Tianjin Medical University

Xin Wang ( $\square$ wx007146@163.com )

Department of General Surgery, The Fourth Center Hospital, Tianjin,China; Division of Bioengineering, Department of Regenerative Medicine, The Fourth Center Hospital, Tianjin, China.Key Laboratory of Hormones and Development (Ministry of Health), Key Laboratory of Metabolic Diseases, Tianjin Metabolic Diseases Hospital. Institute of Endocrinology, Tianjin Medical University, Tianjin,China. https://orcid.org/0000-0002-6416-953X

\section{Research}

Keywords: pancreas, acellular pancreatic bioscaffold, Bone mesenchymal stromal cells, proliferation ,differentiation, chronic pancreatitis

Posted Date: August 17th, 2020

DOI: https://doi.org/10.21203/rs.3.rs-59795/v1

License: (c) (1) This work is licensed under a Creative Commons Attribution 4.0 International License. Read Full License 


\section{Abstract}

Background: To evaluate the potential differentiation ability of bone mesenchymal stromal cells(BMSCs) to pancreatic line Cells on rat acellular pancreatic bioscaffold(APB) and the effect of differentiated BMSCs for chronic pancreatitis(CP) in vivo.

Methods: After BMSCs were isolated and identified, they were dynamic cultured on the APB and static cultured in tissue culture flask(TCF), with or without the growth factors (GF) in both the culture system. The cytological behavior such as the proliferation and differentiation of BMSCs in all the above kinds of culture system were assessed by morphological observation, flow cytometry, ELASA analysis, qRT-PCR assay and western blot analysis. For the in vivo study, the pancreatic fibrosis and pathological score were evaluated. And also the expression of a-SMA, collagen type I and III, IL-10 in pancreas tissue were detected by ELASA.

Results: $4 \mathrm{ml} / \mathrm{min}$ was the most appropriate flow rate for the dynamic culture of BMSCs. The proliferation rate of BMSCs in the APB groups were significantly increased compared to TCF system. During the pancreatic line cell differentiation process, APB could induce BMSCs express markers such as PDX-1 and PTF-1 at higher mRNA levels. In contrast, the marker Oct 4 was expressed at a lower level in APB group. For the pancreatic functional cytoketatins including a-Amy, CK7, Flk-1, and C-peptide, they were all expressed at higher level in APB group. And metabolic enzymes secretion such as amylase and insulin were promoted significantly in APB system. By scanning electron microscope(SEM) and transmission electron microscopy(TEM), the ultrastructure of BMSCs in the APB group could further demonstrated the morphological characteristics of pancreatic-like cells. In vivo study,the expression of a-SMA, collagen type I and III in tissues were less in differentiated BMSCs treatment group, while the level of IL-10 in pancreatic tissue were higher in differentiated BMSCs treatment group with significant difference $(P<0.05)$. In addition, in both in vitro and in vivo study, GF could significantly facilitate the function of proliferation, differentiation and pancreatic cell therapy.

Conclusion: Together our data show the capacity of APB , 3D pancreatic biomatrix, promoting BMSCs differentiate toward pancreatic line phenotypes, and the considerable potential of using these cells for pancreatic cell therapies and tissue engineering.

\section{Introduction}

Severe pancreatic disease such as severe acute pancreatitis and pancreatic cancer may cause pancreatic failure, which poses a serious harm to human health, with an exceedingly high global mortality rate[1-4]. As there are lack of effective therapies, pancreas transplantation has been proposed as a potential therapeutic alternative for the treatment of organ defection or tissue injury. As organ transplantation has been greatly limited by the imbalance between demand and supply of suitable donor organs[5], regenerative medicine(RM) being an interdisciplinary and a very attractive field of research seeks to 
overcome the limitations of replacement and transplantation treatment by facilitating the natural development of tissue[6].

One of the roadblocks to success in RM field is the identification of cells that can be used to regenerate bioengineered organs[7-8]. Stem cells and their descendants or committed progenitors, are capable of proliferating and differentiating into specialized cells[9]. Due to their ability to self-renew and maintain indefinitely a population with identical properties through symmetric and asymmetric cell divisions, stem cell therapies for diseased solid organs are an important potential modality of RM[10].

Mesenchymal stem cells(MSC) can be isolated from tissues such as bone marrow, adipose tissue, umbilical cord tissue or amniotic fluid. Due to their characteristics such as self-renewal and multilineage differentiation capability into osteogenic, adipogenic, chondrogenic, and myogenic-and neurogenic-like lineages [11-15], MSCs offer great therapeutic potential and have been developed to treat a wide range of disorders.

The most extensively studied MSC is bone mesenchymal stromal cells(BMSCs), characterized as fibroblast-like cells, which are isolated from the bone marrow mesenchymal cellular populations. BMSCs are renowned in RM for their multilineage differentiation potential and easy acquisition[15,16]. Aside from their remarkable proliferative and multilineage differentiation/ regenerative potential, BMSCs can affect the surrounding microenvironment by their multiple impressive paracrine functions $[17,18]$. Also, they have immunomodulatory and antioxidant properties[19]. Since the introduction of cell therapy as a strategy for the treatment of many diseases, BMSCs have emerged as ideal candidates.

BMSCs can be differentiate into cells of pancreatic lineages under certain culture system[20]. But such inducing stratergy can not provide conditions for BMSCs proliferating rapidly with high viability. Also, these kinds of induced BMSCs can not maturely express the important pancreatic lineages cells markers. They will loss of stem cell characteristics and functions after expansion in vitro[21,22].

Acellular matrix(ACM) plays an ideal three-dimensional(3D) platform for regenerative medicine because it is biocompatible and preserves 3D geometric and spatial architecture[23]. ACM has physiological levels of biochemical components, matrix-bound growth factors, and cytokines[24]. Furthermore, ACM had intact and patent vasculature structures which could transport nutrition and oxygen for seeding cells to attach and localize to specific topographical position[25].

Previous study indicated that certain kinds whole organ ACM could support BMSCs differentiating into mature cells and expressing functional markers[26-29]. Furthermore, this type of induced cells had potential applications in regenerative therapy and tissue repair[30]. But less research previously have been shown about whole organ ACM promoting BMSCs pancreatic differentiation.

Past work from our laboratory indicated the biological utility of acellular pancreatic biomaterial scaffolds(APB) as whole organ ACM, which could support and enhance AR42J pancreatic acinar cells proliferation and differentiation for regenerative medicine[31].Therefore, in this study, we determine to 
investigate the ability of APB to promote BMSCs proliferation and differentiation which may improve regenerative therapy.

\section{Materials And Methods}

\section{Isolation and identifcation of BMSCs}

All animal work was approved by the Institutional Animal Care of China and performed in accordance with the Animal Welfare Act Institutional Guidelines. BMSCs were collected from the bone marrow of the Adult Sprague Dawley rats (weighing approximately $250 \mathrm{~g}$ between 6 and 7 weeks old). The rats were euthanized by chloroform, and the femur and tibia were removed. In order to collect BMSCs, a 23-gauge syringe was inserted into the bone cavity and flashed with serum-free Dulbecco's modified Eagle's medium (DMEM). After centrifuging at $1200 \mathrm{rpm}$ for $10 \mathrm{~min}$, the bone marrow cells were re-suspended in DMEM(HyClone,USA) that was supple-mented with $10 \%$ fetal bovine serum (FBS), $10 \mathrm{U} / \mathrm{ml}$ penicillin, $10 \mu \mathrm{g} / \mathrm{ml}$ streptomycin (Gibco, Australia), and 1\% L-glutamate(Sigma,USA). Finally, the number of viable cells was checked and transferred to culture dishes at a density of $5 \times 10^{5}$ cells per $\mathrm{cm}^{2}$ in high-glucose DMEM containing $10 \%$ FBS for incubation in $37^{\circ} \mathrm{C}$ and $5 \% \mathrm{CO} 2$ atmosphere. The medium was changed every 2 to 3 days. Only low-passage $(\leq 5)$ cells were used in experiments. Cells were passaged every 7-10 days at a 1:3 ratio. BMSCs were characterized by flow cytometry for expression of CD90, CD29, and CD45.

\section{Decellularization of rat pancreas and reseeding of APB}

Adult Sprague Dawley rats, weighing approximately $250 \mathrm{~g}$ between the ages of 6 to 7 weeks, were anesthetized. As previous study described, the pancreata were decellularized by Easy-Load Digital Drive peristaltic pumps and the biocompatibility of APB was assessed[31,32]. BMSCs were reseeded onto APB and cultured in the biomimetic bioreactor system made by our laboratory for 7 days in order to evaluate repopulation and further differentiation. Cells were seeded on APB by multi-step infusion with $2.5 \times 10^{6}$ in $2 \mathrm{ml}$ each through hepatic portal vein and pancreatic duct. Medium was changed every 2-3 days. The ultrastructure of seeded BMSCs on APB were observed by scanning electron microscope(SEM) and transmission electron microscopy(TEM). Additionally, to assay the optimal flow rate APB supporting the proliferation of BMSCs, the process of dynamic culture was perfused retrogradely at different speed $(0$, $0.5,1,2,4,6 \mathrm{ml} / \mathrm{min})$. The DNA content of BMSCs in the APB scaffold in each speed was quantified on day 4 and 7 .

\section{The characteristic of BMSCs by laser scanning confocal microscopy(LSCM)}

Tissue samples were fixed in 4\%formaldehyde (ThermoFisher,Waltham,Mass), cryo-protected with 30\% sucrose, and cut into $5 \mu \mathrm{m}$ thick sections. For immunostaining, the rabbit primary antibodies $(1: 200$; Boersen, China) were used. Goat antirabbit Alexafluor 488 (1:500; Invitrogen,USA)was used as a secondary antibody. For co-labeling using antibodies from the same host species, sequential staining by GFP(BioHermes,USA) was conducted. After the first primary antibody staining, an additional blocking 
step was included before the addition of a secondary antibody. The slides were washed three times with 1×PBS(5-10 minutes each) before being mounted with ProLong Gold Anti-fade Reagent with DAPI (Invitrogen). After washing,the cells were incubated with streptavidin-conjugated Texas red (Tx-R) for $30 \mathrm{~min}$ at $37^{\circ} \mathrm{C}$, washed 6 times (10min each) with PBS and mounted on glass slides using FITC-guard (Testog Inc., IL) as the mounting medium.

Cells were then examined in a PHOIBOS 1000 laser scanning confocal microscope (Sarastro,Stockholm, Sweden). Tx-R was excited with an argon laser. The emitted signals were collected and used to create three-dimensional reconstructions of serial confocal sections using the programVanis(Sarastro, Stockholm, Sweden).

\section{In vitro differentiation of BMSCs into pancreatic line cells}

After BMSCs reseeding on APB for 7 days, they were induced into pancreatic-like clusters in the differentiation culture system ibcluding dynamic cultured on the APB and static cultured in tissue culture flask(TCF) in 3 steps for another 21 days. The time at the end of the $7^{\text {th }}$ day of BMSCs reseeding on APB was recorded as time zero. Step1:BMSCs were cultured in high glucose DMEM $(25 \mathrm{mmol} / \mathrm{L})$ containing $2 \%$ FBS, $0.2 \mathrm{mmol} / \mathrm{L} \beta$-mercaptoethanol(Gibco), 10ng/ml bFGF(Peprotech,USA ) and 10ng/ml EGF(Peprotech) for 7 days. Step two: BMSCs were cultured in serum free high-glucose DMEM (25mmol/L) containing 10ng/ml bFGF, 10ng/ml EGF, 2\% B27(Gibco), 0.5\% BSA, 10mmol/L nicotinamide(Sigma)and 10ng/ml exendin-4(Sigma)for 7 days. Step three: The cells were cultured in serum-free high-glucose DMEM (25mmol/L) containing 10ng/ml EGF, 10ng/ml Activin A(Peprotech), 10ng/ml betacellulin (Peprotech), 2\% B27 and 0.5\% BSA for 7 days. The medium was changed every 2 days. For TCF static culture system, cells were cultured in TCF system with no flow rate,while in APB dynamic system, cells were cultured in the biomimetic bioreactor system on APB in the optimal flow speed. Based on whether the differentiation was induced by the growth factors (GF) in the culture system, our study was divided into 4 groups: BMSCs cultured in TCF without any GF(TCF-GF(-)), BMSCs cultured in TCF with GF(TCF-GF(+)), BMSCs cultured on APB without GF(APB-GF(-)), BMSCs cultured on APB with GF (APB-GF(+)).

\section{The morphological characteristic of BMSCs in differentiation by SEM and TEM}

The samples were fixed in $2.5 \%$ glutaraldehyde in $0.1 \mathrm{M} \mathrm{PBS}(\mathrm{pH} 7.4)$ for 60 minutes, washed thoroughly in 3 changes $0.1 \mathrm{M}$ PBS for 15 minutes each. Next, the samples were fixed in $1 \%$ OsO4 in $0.1 \mathrm{M}$ PBS for 60 minutes. This was followed by another 3 changes of PBS washing steps for 15 minutes each. The samples were then dehydrated in gradient series of alcohol for $15 \mathrm{~min}$ each. Additionally, samples were critical point dried and coated with Au/Pd using a Cressington Coater 108A sputter coater. Electron microscope images were taken using a Jeol JSM-6335F field emission SEM.

For TEM, the sample was fixed in $2.5 \%$ glutaraldehyde in PBS.Then were post-fixed in $1 \%$ osmium tetroxide in PBS, dehydrated through a graded series of alcohols and embedded in Epon. Thin (60-nm) sections were cut using a Reichert Ultracut S, mounted on 200 mesh copper grids and counterstained 
with $2 \%$ aqueous uranylacetate for 7 min and $1 \%$ aqueous lead citrate for 2 min. Observation was with a JEOL 1011 TEM.

\section{Cell Proliferation Assay}

Bromodeoxyuridine (BrdU) incorporation was analyzed immunohistochemically using a BrdU immunohistochemistry system. Cell proliferation was also assessed by measuring the BrdU incorporation using a commercially available BrdU ELISA kit(Abcam,UK) according to the manufacturer's protocol. Cellswere fixed by a fixation solution and incubated with anti-BrdU antibody for 90 minutes. After washing, tetramethyl-benzidine was added, and absorbance was measured by a spectrophotometric plate reader at 405-nm wavelength.

\section{The expression of pancreatic line gene markers assessment by RT-PCR}

RNAwas extracted using a NucleoSpin kit (Seebio Biotech,China) according to the manufacturer's protocol. The absorbance at 280 and $260 \mathrm{~nm}$ was measured using a BioRad Smart Spec spectrophotometer (Biorad Laboratories, USA) to evaluate the RNA concentration and quality. Reverse transcription was performed using the ImProm II (Promega, Madison,Wis) reverse transcription kit according to the manufacturer's recommendations.Quantitive real time-polymerase chain reaction analysis was performed for pancreatic acinar genes. Analysis was repeated in triplicate.

\section{Quantification of pancreatic functional cytoketatins by western blot}

Whole cell lysates were prepared to evaluate important pancreatic proteins. Antibodies were used at the following concentrations: anti-a-Amy, 1:1000; anticytokeratin 7 (CK7), 1:2500; C-peptide, 1:2500 and antifetal liver kinase-1 (Flk-1), 1:2000. Membranes were incubated with the appropriate IgG HRP-conjugated secondary antibody (1: 5000). Antibody binding was detected by chemiluminescence radiography. Membranes were scanned, recorded digitally, and processed using Image $J$ software.

\section{Quantification of potential metabolic function of differentiated BMSCs in vitro}

For the insulin release assay in vitro, the cell clusters were tested using four different glucose concentrations $(5,10,15$ and $25 \mathrm{mmol} / \mathrm{L})$. The sample were pre-incubated with Krebs-Ringer bicarbonate (KRB) buffer for $1 \mathrm{~h}$. After that, the clusters were removed and incubated for $1 \mathrm{~h}$ in KRB containing different concentrations of glucose.The supernatant was collected and frozen at minus $80^{\circ} \mathrm{C}$. Insulin levels were then measured using rat insulin enzyme-linked immunosorbent assay (ELISA) kits (Mercodia, Sweden) according to the manufacturer's protocol.

On the other hand, amylase(Amy) secretion were measured by 4 different cholecystokinin (CCK) including $0 \mathrm{mmol} / \mathrm{L}, 10^{-9} \mathrm{mmol} / \mathrm{L}, 10^{-8} \mathrm{mmol} / \mathrm{L}, 10^{-7} \mathrm{mmol} / \mathrm{L}$. The values for amylase are expressed as ratios between the amount of amylase released into the extracellular medium and the total cellular amylase determined by permeabilizing cells with $0.1 \%$ SDS in $10 \mathrm{mM}$ phosphate buffer ( $\mathrm{pH} 7.8)$. The metabolic 
enzymes Amy was spectrophotometrically using Phadeba amylase kit according to the manufacturer's protocol. Both the above 2 process were performed at the end of differentiation process.

\section{In vivo performance studies of differentiated BMSCs}

To investigate the effects of differentiated BMSCs for treating chronic pancreatitis(CP) in vivo, our research were randomly divided into 6 groups with 10 rats each: control group, model group and 4 treatment group (BMSCs cultured on 4 different system: TCF-GF(-), TCF-GF(+), APB-GF(-), APB-GF(+)). CP rat model was induced by infusion of dibutyltin dichloride (DBTC) through the caudal vein. For the treatment group, differentiated BMSCs were injected through caudal vein at the volume of $3 \times 106 / \mathrm{mL}$ in $1 \mathrm{ml}$ at 20 days after the model induction and the injection was repeated twice at 27 days and 34 days after model induction.For the model group, the equal volume of saline was injected into the caudal vein.

For all the rats, pancreatic tissues were collected 80 days after model induction for histopathological examination and the expression of a-SMA, collagen type I and III, IL-10 detection by ELASA .

\section{Statistical Analysis}

Data were expressed as means (standard deviation [SD]). Significant differences among groups were determined by the t-test for 2-group comparisons and analysis of variance of repeated measure followed by post hoc analysis for multiple group comparisons.Probability values at $\mathrm{P}<0.05$ indicated statistical significance.

\section{Results}

\section{Characterization of BMSCs isolated from bone marrow}

The morphology of BMSCs in Passage 1 exhibited irregular round shape and size(Figure 1a) while Passage 3 of BMSCs changed to regular form and showed a spindle fibroblast-like appearance(Figure 1b). Early passage of BMSCs between two and four passages, were $90.21 \%$ positive for both CD90 and CD29, 98.53\% positive for CD90 and negative for CD45, 93.38\% positive for CD29 and negative for CD45.

\section{Repopulation of APB with BMSCs in pancreatic bioreactor cultures}

The seeded BMSCs attached on the surface of the APB bioscaffold and exhibited different shapes (yellow arrows). The adhesion among neighboring cells(white arrows) could be observed by SEM (Figure 2a). Also, LSCM indicated that BMSCs not only attached on the surface of the bioscaffold,but also formed in cluster in the inner structure of APB.The three-dimensional scaffolds reseeded with the GFPpositive cells(green) were counterstained with component collagen(red) and DAPI(blue) (Figure 2b). The APB, as the three-dimensional scaffolds,could support the reseeding of BMSCs.

Also,the DNA quantification of the BMSCs-APB graft showed that $4 \mathrm{ml} / \mathrm{min}$ for the dynamic culture system was the most appropriate flow rate for BMSCs proliferation in both day 4 and day 7 with 
significant difference compared to other flow speed $(P<0.05)$.(Figure 3$)$

\section{The ultrastructural characteristics of BMSCs during differentiation process}

The undifferentiated BMSCs indicated spindle fibroblast-like appearance with little microvilli at the surface of the cells by SEM (Figure 4a). There was not any mature organelle by TEM (Figure 4b,4c).

The differentiated BMSCs at the 21st day was spherical in shape with more dense microvilli at the surface of the cells by SEM(Figure 5a). There was a major increase number of epithelial-like cell clusters(white arrow) with a complex intercalation of the extracellular matrix(ECM) (black arrow) (Figure $5 b)$. Differentiating endocrine and exocrine cells developed from the BMSCs and subsequently formed isolated small clusters(Figure $5 \mathrm{c}$ ). A significant amount of extracellular matrix fibers accumulated around each newly differentiated cells as well as the cell clusters (Figure $5 \mathrm{~d}$ ).

By TEM, pancreatic-like epithelial cells(white arrow)were organized into ductal structures (black arrow)surrounded by scattered individual mesenchymal-like stellate cells(Figure 5e).The epithelial cells had formed small clusters separate from the ducts(black arrow). Acinar cells increased in number and formed amylase cell clusters(red arrow). The cell clusters for insulin had also increased in numbers and formed islet-like structure(white arrows)that contained capillaries(yellow arrows)(Figure 5f).

Also,TEM showed glycogen positivity in ductal cells(black arrows), nucleus(blue arrows), secretory granules(yellow arrow), mitochondria(white arrow), smooth endoplasmic reticulum and rough endoplasmic reticulum(red arrow) in differentiated BMSCs(Figure $5 \mathrm{~g}$ ).

\section{BMSCs proliferation in the differentiation process among 4 groups}

To determine whether the APB allowed BMSCs to grow and to compare its efficacy with other kinds of culture system(Figure 6), BrdU incorporation of BMSCs proliferated with time-dependent growth from day 3 to 21 in all 4 groups. From day 3, the cell proliferation rate was significantly higher in the APB dynamic system than in the TCF culture with or without $G F(P<0.05)$. Also, at the present of $G F$, the proliferation rate was higher in both APB system and TCF system with significant difference from day $3(P<0.05)$. These results suggest that the APB efficiently facilitates pancreatic-like cell growth and GF could promote BMSCs proliferation.

\section{Evaluation of the expression of pancreatic gene markers}

To assess the ability of the APB to support BMSCs differentiation, the expression of pancreatic genes at different time points of all the culture system was assessed (Figure 7). The PDX-1 and PTF-1 gene increased while Oct 4 gene decreased from day 3 to 21 for all samples. Furthermore, in both 2 kinds of culture system, the PDX-1 and PTF-1 gene were higher while the Oct4 was less in GF(+) groups compared with GF(-) groups. In addition, with or without GF, the expression of PDX-1 and PTF-1 was higher while Oct4 was lower for BMSCs cells cultured on the APB groups. The expression of PDX-1 and PTF-1 was higher for cells cultured on the APB-GF(-) system than on TCF-GF(-) system with significant difference 
from day 7, while was higher for cells cultured on the APB-GF $(+)$ system than on TCF-GF $(+)$ system with significant difference from day 3. On the other hand, Oct4 was lower for cells cultured on the APB-GF(-) system than on TCF-GF(-) system with significant difference from day 5 , while was lower for cells cultured on the APB-GF(+) system than on TCF-GF(+) system with significant difference from day 3.

\section{Evaluation of the expression of pancreatic cytoketatins}

To verify these findings, we performed western blotting of pancreatic cytoketatins(Figure 8). The protein expression of a-Amy, CK7, C-peptide and Flk- 1 was increased from day 3 to 21 for all groups. Also, in both 2 kinds of culture system, such four cytoketatins were higher in GF(+)groups compared with GF(-)groups. In addition, with or without GF, the expression of the 4 cytoketatins was higher for BMSCs cells cultured on the APB than for cells in TCF group. The expression of C-peptide was higher in APB-GF(-) group than in TCF-GF(-) group with significant difference from day 5, while was higher in APB-GF $(+)$ group than in TCF$\mathrm{GF}(+)$ group with significant difference from day 3 . For other 3 kinds of cytoketatins, the expression was higher in APB-GF(-) group with significant difference from day 14, while was higher in APB-GF(+) group with significant difference from day 5.The gene and cytoketatin results suggest that pancreatic cell differentiation is enhanced in the presence of APB and GF could promote BMSCs differentiation..

\section{Assessment of pancreatic metabolic function of differentiated BMSCs}

The insulin secretion levels $(\mathrm{pg})$ of cell clusters were detected in the presence of 5, 10, 15 and 25mmol/L glucose(Figure 9a). The insulin levels enhanced when concentrations of glucose increased in all 4 groups. At the lowest concentration of $5 \mathrm{mmol} / \mathrm{L}$, there were no significant difference among 4 groups $(P>0.05)$. At the concentration of $10 \mathrm{mmol} / \mathrm{L}$, there were significant difference at the presence of GF in both dynamic system and static system $(P<0.05)$, but still no significant difference between dynamic system and static system with or without $G F(P>0.05)$. At the higher concentrations of 15 and $25 \mathrm{mmol} / \mathrm{L}$ glucose, with or without GF, insulin secretion levels were higher in the APB groups than in TCF groups $(P<0.05)$, and the level of insulin were significantly higher at the presence of GF in both APB and TCF culture system $(P<0.05)$.

The amylase secretion in the culture system were detected in the presence of $10^{-11} \mathrm{mmol} / \mathrm{L}, 10^{-10} \mathrm{mmol} / \mathrm{L}$, $10^{-9} \mathrm{mmol} / \mathrm{L}$ and $10^{8} \mathrm{mmol} / \mathrm{L}$ CCK (Figure $9 \mathrm{~b}$ ). The amylase levels enhanced when concentrations of CCK increased in all 4 groups. At the concentration of $10^{-11} \mathrm{mmol} / \mathrm{L}, 10^{-10} \mathrm{mmol} / \mathrm{L}$, there were no significant difference among 4 groups $(P>0.05)$. At the concentration of $10^{-9} \mathrm{mmol} / \mathrm{L}$ and $10^{-8} \mathrm{mmol} / \mathrm{L}$, there were significant difference between APB system and TCF system with or without $G F(P<0.05)$, and also the amylase secretion levels were significantly higher at the presence of GF in both APB and TCF culture system $(P<0.05)$.

\section{Investigation of differentiated BMSCs treatment for CP in vivo}

Both the pathological score and the pancreatic fibrosis score(table 1) were lower in each of the treatment group with significant difference compared with model group $(P<0.05)$. In the 4 treatment group, with or 
without GF, the pathological score and the pancreatic fibrosis score were lower in APB group than in TCF group with significant difference $(P<0.05)$. In addition, in both 2 kinds of culture system, such 2 score were higher in GF(-)groups compared with $G F(+)$ groups with significant difference $(P<0.05)$.

The expression of a-SMA, collagen type I and III in tissues (table 2) were less in each of the treatment group compared with model group with significant difference $(P<0.05)$, while the level of IL-10 in pancreatic tissue were higher in treatment group compared with model group with significant difference $(P<0.05)$. There were no significant difference for the expression of a-SMA, collagen type $I$, collagen type III between treatment group and control group $(P>0.05)$. In the 4 treatment group, with or without $G F$, the expression of a-SMA, collagen type I and III were lower while the level of IL-10 were higher in APB group than in TCF group with significant difference $(P<0.05)$. In addition, in both 2 kinds of culture system, the expression of a-SMA, collagen type I and III were lower while the level of IL-10 were higher in GF(+)groups compared with GF $(-)$ groups with significant difference $(P<0.05)$.

\section{Discussion}

For purposes of tissue regeneration either in vivo or in vitro, it is essential for the donor cell including the following characteristics (1) the ability to differentiate into pancreatic cell types, (2) high proliferative potential(must be able to expand to high numbers prior to seeding onto the APB) (3) be easily accessible(an autologous cell source,either differentiated or stem), and (4) lack of immunogenicity. Such the above characteristics point to the possibility of using BMSCs as a source for reseeding on APB or as in vivo cell therapy.

The growth of cells should be regulated by their microenviroment around including the attachment among cells and the signal molecules.It is important for cells to proliferate and differentiate in culture system mimicing the microenvironment of their innate tissue which can not be accomplished by traditional 2D culture system[33,34]. ACM with preserved complex multifaceted ECM composition, 3D spatial orientation and microstructure, should have tissue-specific biological nature as well as biocompatible properties that can promote cell adhesion, viability, proliferation, and differentiation[35]. Also, ACM has physiological levels of growth factors such as insulin-like GF, bone morphogenetic protein 4 and cytokines for regeneration and implantation[36,37].This can explain why the APB have the ability of prompting BMSCs differentiation.

Furthermore, appropriate flow rate is better for BMSCs proliferation and differentiation in the dynamic culture system than static culture in APB. Dynamic culture is benefit for $\mathrm{O} 2$ and nutrient delivery which are necessary for BMSCs. Also, the metabolic waste around BMSCs can be easily removed in flow culture system[38]. In addition, the flow speed in the dynamic culture system can generate liquid shearing force which will appropriately modulated BMSCs proliferation and differentiation. Lowering or enhancing the flow rate will led to the increase of BMSCs cell apoptosis proportion[39].

In our study, BMSCs could be reseeded and repopulation on the surface and in the center of APB by morphological assessment. Also, BMSCs could survive and proliferate with high viability at $4 \mathrm{ml} / \mathrm{min}$ for 
BMSCs proliferation in the dynamic culture system. $4 \mathrm{ml} / \mathrm{min}$ will be utilized as the most optimal flow rate in our study.

For the purpose of BMSCs differentiation, the GF was added in both 2 kinds of culture system. During the differentiation process, BMSCs could proliferate with time-dependent growth in all 4 groups. The cell proliferation rate was higher in the APB dynamic system than in the TCF culture system. The proliferation rate was higher in APB-GF(+)system than in the APB-GF(-) system. Such results indicated that the APB can efficiently facilitates pancreatic-like cell growth and GF could promote BMSCs proliferation during differentiation process.

For ultrastructural characteristics of BMSCs through differentiation process, the shape of cells had been transferred into spherical with more dense microvilli. Both endocrine and exocrine cells increased in number and formed cell epithelial-like clusters with significant amount of extracellular matrix fibers accumulated around. Also, glycogen positivity, nucleus, secretory granules and mature organella such as mitochondria, smooth endoplasmic and reticulum rough endoplasmic reticulum had been clearly showed in our study. This indicates that BMSCs can be differentiated into pancreatic-like cells. Study has indicated that cell aggregation was a necessary condition for the BMSCs differentiation[40]. This result is in accordance with our currentresearch.

To assess pancreatic differentiation, the expression of genes PDX-1 and PTF-1 that were known to be important to pancreatic function were evaluated. PDX-1 plays a vital role in pancreatic development and differentiation[41]. PTF-1, as transcriptional regulation of exocrine-specific genes and exocrine transcription factor, is responsible for pancreatic exocrine function and exocrine gene expression[42]. In our research, without GF, PDX-1 and PTF-1 were higher on the APB system than on TCF system with significant difference from day 7,while were higher with significant difference from day 3 with GF. This indicates that APB can support BMSCs differentiation and GF can increase such function of APB.

On the other hand, Oct4, the pluripotency markers which associated with the differentiation potential of BMSCs [43], decreased after differentiation. Oct4 was lower for cells cultured on the APB system than on TCF with significant difference after day 5 without GF, but lower with significant difference from day 3 with GF. The expression of genes PDX-1, PTF-1 and Oct4 indicates that APB can promote BMSCs reducing its pluripotency capacities and differentiate into pancreatic like cells especially under the help of GF.

Also, to identify the pancreatic-like cells, we also assessed the expression of the cytoketatins such as aAmy, CK7, C-peptide and Flk-1. a-Amy is a well-established marker of pancreatic acinar cells. Both CK7 and Flk-1 are localized normally in the pancreatic duct structures in the adult pancreas and contribute to the formation of the large intralobular, the interlobular, and the main duct[44]. Coupled with CK7, Flk-1 functions as the receptor of vascular endothelial growth factor, which is associated with the endothelial layer of the vasculature[ 45,46$]$.These two markers are known to be expressed during pancreatic morphogenesis in the fetus[47]. C-peptide is expressed in pancreatic endocrine cells which indicate $\beta$-cells function[48]. 
Our study indicated that the expression of a-Amy, CK7, and Flk-1 was significantly higher for BMSCs cells cultured on the APB than for cells in TCF group after day 14 without GF,while was higher with significant difference from day 5 with GF. Also, C-peptide was higher for cells cultured on the APB system than on TCF with significant difference after day 5 without GF, but higher with significant difference from day 3 with GF. The cytoketatin results suggest that BMSCs could differentiatied into two major pancreatic line cell types:1) the endocrine cells which arrange mainly in groups as islets of Langerhans and secrete different polypeptides delivered to other parts of the body via the vasculature; 2) the secretions of acinar exocrine cells carry away through the ductal system. And GF can strengthen BMSCs induction on APB.

Research had indicated that the mechanical characterization such as the stiffness of biomechanical strength of ACM was relative with BMSC differentiation through TGF- $\beta$. Also, the changing extracellular matrix density inhibits capillary morphogenesis and neovascularization

in vivo in a manner consistent with that observed in vitro[49,50]. Such maybe another mechanism about the inductive effect of the APB

Further study also assessed the metabolic function of differentiated BMSCs. To demonstrate the endocrine function, we compared the insulin secretion levels at different glucose concentrations. The insulin levels enhanced when concentrations of glucose increased. Insulin secretion levels were significantly higher in the APB groups than in TCF groups at the higher concentrations of 15 and $25 \mathrm{mmol} / \mathrm{L}$ glucose. GF could significantly increase insulin secretion. Such trend was not significant at the low concentration of glucose.

Similarly, the amylase levels enhanced when concentrations of CCK increased. At the higher concentration of CCK in $10^{-9} \mathrm{mmol} / \mathrm{L}$ and $10^{-8} \mathrm{mmol} / \mathrm{L}$, Amy was significantly higher in APB system than in TCF system. Amy secretion could significantly increase at the presence of GF. Low level of CCK could not show such trend. Studies had indicated that CCK at less than $10-8 \mathrm{mmol} / \mathrm{L}$ could stimulate Amy secretion in concentration dependent,while CCK more than

$10^{-8} \mathrm{mmol} / \mathrm{L}$ might inhibit Amy secretion[51]. We uitilized CCK less than $10^{-8} \mathrm{mmol} / \mathrm{L}$ in our study.

The assay of metabolic function indicates that APB with GF can promote the pancreatic organogenesis of BMSCs and support BMSCs differentiated into primary functional units which maintain their respective phenotypic expression (endocrine $\beta$-cells: insulin and exocrine acinar cells: Amylase)-in close proximity to their respective native niches.

As whole organ ACM had physiological resemblance of the original tissue, including intact 3D architecture, preserved native ECM components, vascular networks, and biomechanical properties, it could guide tissue regrowth and encourage cell differentiation when combined

with biological agents[52]. Our study is in consists with previous study, 
Previous study has indicated that BMSCs were less immunogenic for they did not express MHC class II markers, and did not elicit a strong immune response as evidenced by lack of activation of T cells[53]. Additionally, BMSCs was easy acquisition and easily accessible. Based on all the above merits of BMSCs, our findings will support in vivo cell therapy efforts, as well as in vitro treatments in whole-pancreas regeneration.

A pathological hallmark of CP is progressive fibrosis that is mediated by the PSCs. One of the earliest cellular events at the initiation of fibrosis is activation of PSCs, which can be mediated concomitantly by a variety of factors, such as oxidative stress, cytokines, growth factors. The process of PSC activation is marked expression of alpha-smooth muscle actin (a-SMA), which can promote the secretion of collagen type I, collagen type III [54,55]. IL-10 has been indicated to be a protective cytokine and has been shown to decrease the severity of chronic pancreatitis and to reduce the likelihood of systemic complications. Also,it can downregulate collagen synthesis in both unstimulated and activated PSC[56,57].

Our study indicated that the expression of PSCs, collagen type I and collagen type III could be downregulated while the expression of IL-10 could be upregulated by differentiated BMSCs in vivo.This indicated that differentiated BMSCs could inhibit the process of fibrosis after transplantation in vivo and have the protective effect for pancreatic tissue.

It should be emphasized that there are several unavoidable limitations of this study. Firstly, the cells after BMSCs differentiation in our research do not have intact properties and function of native phenotypic pancreatic cells. We have to improve our differentiation strategy in further research. Secondly, the article is based on animal model. In the future study, we will provide opinions/insights regarding the feasibility and usefulness of bioscaffolds and cells in humans. Thirdly, our study focuses on APB and BMSCs used in vitro. The efforts of supporting pancreatic tissue engineering in vivo will be provided in future research.

Despite these limitations, we believe that our data truly represented valuable and reliable information related to APB and BMSCs.

\section{Conclusions}

APB could support the proliferation and viability of BMSCs in dynamic culture with optimal flow rate $4 \mathrm{ml} / \mathrm{min}$. During differentiation process, APB could induced BMSCs into pancreatic-like cells which expressed gene markers and pancreatic functional cytoketatins, and promote metabolic enzymes secretion. GF could significantly facilitate the function of proliferation, differentiation and cell engraftment in APB.Our study had the considerable potential for pancreatic cell therapies and pancreatic tissue engineering.

\section{Abbreviations}

BMSCs: bone mesenchymal stromal cells MSC: Mesenchymal stem cells 
APB: acellular pancreatic bioscaffold ACM: Acellular matrix

CP: chronic pancreatitis

TCF :tissue culture flask

GF: growth factors

SEM: scanning electron microscope

TEM: transmission electron microscopy

LSCM: laser scanning confocal microscopy

PDX: pancreatic duodenal homeodomain containing transcription factor

PTF: pancreatic exocrine transcription factor

Oct: octamer-binding transcription factor

a-Amy: a-Amylase

CK7\ cytokeratin 7

Flk-1: fetal liver kinase-1

ECM: extracellular matrix

CCK: cholecystokinin

\section{Declarations}

\section{ACKNOWLEDGMENT}

The authors thank Department of Public Health of Tianjin Medical University for the integrity of the data and the accuracy of the data analysis.

\section{Author contributions}

Wang Xin and Li Zhao designed the research; Wang Xin and Du Yue performed the research; Du Yue contributed new reagents/analytic tools; Wang Xin and Li Zhao analyzed the data and wrote the paper.

\section{Conflict-of-interest statement}

There is no conflict of interest to declare. 
Ethics approval and consent to participate:All animal work was approved by the Institutional Animal Care of China and performed in accordance with the Animal Welfare Act Institutional Guidelines.

Consent for publication: Not applicable

Availability of data and materials: Data sharing not applicable to this article as no data-sets were generated or analyzed during the current study

Competing interests: The authors declare that they have no competing interests.

Funding: National Science Foundation(81502509), National Science Foundation of Tianjin City(2014KY04)

\section{Author contributions:}

Wang Xin and Li Zhao designed the research; Wang Xin and Du Yue performed the research; Du Yue contributed new reagents/analytic tools; Wang Xin and Li Zhao analyzed the data and wrote the paper.

Acknowledgements:The authors thank Department of Public Health of Tianjin Medical University for the integrity of the data and the accuracy of the data analysis.

\section{Authors' information:}

Zhao Li, Department of Hepatobiliary Surgery, Peking University People's Hospital; Beijing key laboratory of liver cirrhosis and HCC basic research;

YueDu, Department of Public Health, Tianjin Medical University, Tianjin, P. R. China;

Xin Wang, Department of General Surgery, The Fourth Center Hospital, Tianjin,China; Division of Bioengineering, Department of Regenerative Medicine, The Fourth Center Hospital, Tianjin, China. Key Laboratory of Hormones and Development (Ministry of Health), Key Laboratory of Metabolic Diseases, Tianjin Metabolic Diseases Hospital. Institute of Endocrinology, Tianjin Medical University, Tianjin,China.

\section{References}

[1] Ibadov RA, Arifjanov AS, Ibragimov SK, et al. Acute respiratory distress-syndrome in the general complications of severe acute pancreatitis. Ann Hepatobiliary Pancreat Surg. 2019;23 (4):359-364.

[2] Zhou X, Wang W, Wang C,et al.DPP4 Inhibitor Attenuates Severe Acute Pancreatitis-Associated Intestinal Inflammation via Nrf2 Signaling. Oxid Med Cell Longev. 2019 Nov 15;2019:6181754.

[3] Wang X, Shan YQ, Tan QQ, et al. MEX3A knockdown inhibits the development of pancreatic ductal adenocarcinoma. Cancer Cell Int. 2020 Feb 28;20:63. 
[4] Wenhao Luo, Gang Yang, Wentao Luo, et al. Novel therapeutic strategies and perspectives for metastatic pancreatic cancer: vaccine therapy is more than just a theory. Cancer Cell Int. 2020; 20: 66.

[5] Rijkse E, IJzermans JN, Minnee RC. Machine perfusion in abdominal organ transplantation: Current use in the Netherlands. World J Transplant. 2020;10(1):15-28.

[6] Porzionato A, Stocco E, Barbon S, et al.Tissue Engineered Grafts from Human Decellularized Extracellular Matrices: A Systematic Review and Future Perspectives.Int J Mol Sci. 2018;19(12).

[7] Mendez JJ, Ghaedi M, Steinbacher D, et al. Epithelial cell differentiation of human mesenchymal stromal cells in decellularized lung scaffolds. Tissue Eng Part A. 2014;20(11-12):1735-1746.

[8] Kim IG, Ko J, Lee HR, et al. Mesenchymal cells condensation-inducible mesh scaffolds for cartilage tissue engineering. Biomaterials. 2016;85:18-29.

[9] Junyent S, Garcin CL, Szczerkowski JLA,et al. Specialized cytonemes induce self-organization of stem cells. Proc Natl Acad Sci USA. 2020 Mar 17.

[10] Lanzoni G1, Oikawa T, Wang Y,et al. Concise review: clinical programs of stem cell therapies for liver and pancreas.Stem Cells. 2013;31(10):2047-2060.

[11] Mizuno H, Tobita M, Uysal AC.Concise review: Adipose-derived stem cells as a novel tool for future regenerative medicine.Stem Cells. 2012;30(5):804-810.

[12] Lin HT, Otsu M, Nakauchi H. Stem cell therapy: an exercise in patience and prudence.Philos Trans $R$ Soc Lond B Biol Sci.2013;368(1609):20110334.

[13] Ra JC, Shin IS, Kim SH,et al. Safety of intravenous infusion of human adipose tissue-derived mesenchymal stem cells in animals and humans.Stem Cells Dev. 2011;20(8):1297-1308.

[14] Rastegar F, Shenaq D, Huang J,et al. Mesenchymal stem cells: Molecular characteristics and clinical applications. World J Stem Cells. 2010;2(4):67-80.

[15] Wang $Y$, Chen $X$, Cao W,et al.Plasticity of mesenchymal stem cells in immunomodulation: pathological and therapeutic implications. Nat Immunol. 2014;15(11):1009-1016.

[16] Zhou LL, Liu W, Wu YM,et al.Oral Mesenchymal Stem/Progenitor Cells:The Immunomodulatory Masters. Stem Cells Int. 2020;2020:1327405.

[17] Fawzy E-Sayed KM, Elahmady M, Adawi Z,et al. The periodontal stem/progenitor cell inflammatoryregenerative cross talk: A new perspective. J Periodontal Res. 2019;54(2):81-94.

[18] C. Brown, C. McKee, S. Bakshi, et al. Mesenchymal stem cells:cell therapy and regeneration potential. Journal of Tissue Engineering and Regenerative Medicine. 2019;13(9): 1738-1755. 
[19] Ma Z, Song G, Liu D,et al. N-Acetylcysteine enhances the therapeutic efficacy of bone marrow-derived mesenchymal stem cell transplantation in rats with severe acute pancreatitis.

Pancreatology. 2019;19(2):258-265.

[20] Leung PS, Ng KY. Current progress in stem cell research and its potential for islet cell

transplantation. Curr Mol Med. 2013;13(1):109-125.

[21] Li M, Zhang T, Jiang J,et al. ECM coating modification generated by optimized decellularization process improves functional behavior of BMSCs.Mater Sci Eng C Mater Biol Appl. 2019;105:110039.

[22] Yin $H$, Wang $Y$, Sun Z,et al.Induction of mesenchymal stem cell chondrogenic differentiation and functional cartilage microtissue formation for in vivo cartilage regeneration by cartilage extracellular matrix-derived particles. Acta Biomater. 2016;33:96-109.

[23] Salvatori M, Katari R, Patel T, et al. Extracellular matrix scaffold technology for bioartificial pancreas engineering:state of the art and future challenges.J Diabetes SciTechnol.2014;8(1):159169.

[24] Tsuchiya T, Balestrini JL, Mendez J, et al. Influence of pH on extracellular matrix presservation during lung decellularization. Tissue Eng Part C Methods.2014;20(12):1028-1036. [25] Orlando G, Farney AC, Iskandar SS, et al. Production and implantation of renal extracellular matrix scaffolds from porcine kidneys as a platform for renal bioengineering investigations. Ann Surg. 2012;256(2):363-370.

[26] Zhao W, Li J J, Cao D Y, et al. Intravenous injection of mesenchymal stem cells is effective in treating liver fibrosis. World J Gastroenterol,2012,18(10):1048-1058.

[27] Kakabadze A, Mardaleishvili K, Loladze G,et al. Reconstruction of mandibular defects with autogenous bone and decellularized bovine bone grafts with freeze-dried bone marrow stem cell paracrine factors. Oncol Lett. 2017;13(3):1811-1818.

[28] Yin H, Wang Y, Sun Z,et al. Induction of mesenchymal stem cell chondrogenic differentiation and functional cartilage microtissue formation for in vivo cartilage regeneration by cartilage extracellular matrix-derived particles.Acta Biomater.2016;33:96-109. 
[29] Santhakumar R,Vidyasekar P,Verma RS. Cardiogel: a nano-matrix scaffold with potential application in cardiac regeneration using mesenchymal stem cells.PLoS One.2014;9(12): e114697.

[30] Li M, Zhang T, Jiang J, et al. ECM coating modification generated by optimized decellularization process improves functional behavior of BMSCs.Mater Sci Eng C Mater Biol Appl. 2019;105:110039.

[31] Wang X, Li YG, Du Y, et al. The Research of Acellular Pancreatic Bioscaffold as a Natural 3Dimensional Platform In Vitro. Pancreas. 2018;47(8):1040-1049.

[32] Goh SK, Bertera S, Olsen P, et al. Perfusion-decellularized pancreas as a natural 3D scaffold for pancreatic tissue and whole organ engineering.Biomaterials. 2013;34(28):6760-6772.

[33] Gaetani R, Aouad S, Demaddalena LL, et al. Evaluation of different decellularization protocols on the generation of pancreas-derived hydrogels. Tissue Eng Part C Methods.2018 Nov6.

[34] Porzionato A, Stocco E, Barbon S,et al.Tissue-Engineered Grafts from Human Decellularized Extracellular Matrices: A Systematic Review and Future Perspectives.Int J Mol Sci. 2018 Dec 18;19(12).

[35] Ungerleider JL, Johnson TD, Hernandez MJ, et al. Extracellular matrix hydrogel promotes tissue remodeling, arteriogenesis, and perfusion in a rat hindlimb ischemia model. JACC Basic Transl Sci. 2016;1(1-2):32-44.

[36]Crapo P M, Gilbert T W, Badylak S F. An overview of tissue and whole organ decel-

lularization processes[J]. Biomaterials,2011,32(12):3233-3243.

[37] Kniazeva E, Kachgal S, Putnam A J. Effects of extracellular matrix density and me-senchymal stem cells on neovascularization in vivo[J]. Tissue Eng Part A, 2011,17(7-8):905-914.

[38] Hwang N S, Varghese S, Elisseeff J. Controlled differentiation of stem cells. Adv Drug Deliv Rev,2008,60(2):199-214.

[39] Cartmell S H, Porter B D, Garcia A J, et al. Effects of medium perfusion rate on cell-seeded threedimensional bone constructs in vitro. Tissue Eng, 2003,9(6):1197-1203.

[40] Guo Y, Chen S, Xu L,et al. Decellularized and solubilized pancreatic stroma promotes the in vitro proliferation, migration and differentiation of BMSCs into IPCs. Cell Tissue Bank. 2019;20(3):389401.

[41] Park CH, Lee JY, Kim MY, et al. Oligonol, a low-molecular-weight polyphenol derived from lychee fruit, protects the pancreas from apoptosis and proliferation via oxidative stress in streptozotocin-induced diabetic rats.Food Funct. 2016;7(7):3056-3063.

[42] Barlass U, Dutta R, Cheema H,et al. Morphine worsens the severity and prevents pancreatic regeneration in mouse models of acute pancreatitis. Gut. 2018;67(4):600-602. 
[43] Fafián-Labora J, Morente-López M, Sánchez-Dopico MJ,et al. Influence of mesenchymal stem cellderived extracellular vesicles in vitro and their role in ageing. Stem Cell Res Ther. 2020 Jan 3;11(1):13.

[44] Rooman I, Heremans $\mathrm{Y}$, Heimberg $\mathrm{H}$, et al. Modulation of rat pancreatic acinoductal trans differentiation and expression of PDX-1 in vitro. Diabetologia. 2000;43(7):907-914.

[45] Di Rocco G, Tritarelli A, Toietta G, et al. Spontaneous myogenic differentiation of Flk-1-positive cells from adult pancreas and other nonmuscle tissues. Am J Physiol Cell Physiol. 2008;294(2):C604-C612.

[46] Dygai AM, Skurikhin EG, Pershina OV, et al. Role of hematopoietic stem cells in inflammation of the pancreas during diabetes mellitus. Bull Exp Biol Med. 2016;160(4):474-479.

[47] Xue A, Julovi SM, Hugh TJ, et al. A patient-derived subrenal capsule xenograft model can predict response to adjuvant therapy for cancers in the head of the pancreas. Pancreatology. 2015;15(4):397404.

[48] Boughton C, Allen JM, Tauschmann M,et al. Assessing the effect of closed-loop insulin delivery from onset of type 1 diabetes in youth on residual beta-cell function compared to standard insulin therapy (CLOuD study): a randomised parallel study protocol. BMJ Open. 2020;10(3):e033500.

[49] Park J S, Chu J S, Tsou A D, et al. The effect of matrix stiffness on the differentiation of mesenchymal stem cells in response to TGF-beta[J]. Biomaterials. 2011,32(16):3921-3930.

[50] Subramanian K, Owens D J, O'Brien T D, et al. Enhanced differentiation of adult bone marrow-derived stem cells to liver lineage in aggregate culture[J]. Tissue Eng

Part A,2011,17(17-18):2331-2341.

[51] Gukovskaya AS, Gukovsky I, Jung Y, et al. Cholecystokin in induces caspase activation and mitochondrial dysfunction in pancreatic acinar cells. Roles in cell injury processes of pancreatitis. J Biol Chem. 2002;277(25):22595-22604.

[52] Gong T, Heng BC, Lo EC, et al. Current advance and future prospects of tissue engineering approach to dentin/pulp regenerative therapy. Stem Cells. Int. 2016;2016:9204574.

[53] Rasmusson I, Ringdén O, Sundberg B et al. Mesenchymal stem cells inhibit the formation of cytotoxic T lymphocytes, but not activated cytotoxic T lymphocytes or natural killer cells.

Transplantation. 2003;76(08):1208-1213.

[54] Ratnakar R Bynigeri, Aparna Jakkampudi, Ramaiah Jangala, et al. Pancreatic Stellate Cell:Pandora's Box for Pancreatic Disease Biology.World J Gastroenterol. 2017;23(3):382-405.

[55] Hannah M Komar, Gregory Serpa, Claire Kerscher, et al. Inhibition of Jak/STAT signaling reduces the activation of pancreatic stellate cells in vitro and limits caerulein-induced chronic pancreatitis in vivo.Sci 
Rep. 2017;7(1):1787.

[56]Fu-Xiang Yu, Long-Feng Su, Chun-Lei Dai,et al.Inhibition of Pancreatic Stellate Cell Activity by Adipose-Derived Stem Cells. Hepatobiliary Pancreat Dis Int. 2014;14(2):215-221.

[57] Manohar M, Verma AK, Venkateshaiah SU, et al. Pathogenic mechanisms of pancreatitis. World J Gastrointest Pharmacol Ther. 2017; 8(1): 10-25.

\section{Tables}

\begin{tabular}{|lcll|}
\hline \multicolumn{4}{|l|}{ Table 1 The pancreatic fibrosis and pathological score } \\
\hline Group & $\mathrm{n}$ & pathological score & pancreatic fibrosis score \\
\hline Controlgroup & 10 & 0 & 0 \\
\hline Model group & 10 & $9.89 \pm 3.31$ & $2.51 \pm 0.41$ \\
\hline Treatment group & & & \\
\hline TCF-GF(-) & 10 & $7.79 \pm 2.57$ & $0.96 \pm 0.18$ \\
\hline TCF-GF(+) & 10 & $5.84 \pm 2.11$ & $0.78 \pm 0.17$ \\
\hline APB-GF(-) & 10 & $3.91 \pm 1.33$ & $0.61 \pm 0.24$ \\
\hline APB-GF(+) & 10 & $2.11 \pm 0.86$ & $0.37 \pm 0.09$ \\
\hline F & & 3.182 & 4.055 \\
\hline P & & 0.038 & 0.024 \\
\hline
\end{tabular}




\begin{tabular}{|llllll|}
\hline \multicolumn{5}{|c|}{ Table 2 The expression of a-SMA, collagen type I and III, IL-10in pancreatic tissue $(\mathrm{mg} / \mathrm{ml})$} \\
\hline & $\mathrm{n}$ & $\mathrm{a}-\mathrm{SMA}$ & collagen type I & collagen type III & $\mathrm{IL}-10$ \\
\hline Control group & 10 & $70.22 \pm 24.41$ & $42.59 \pm 19.56$ & $230.79 \pm 85.55$ & $2808.11 \pm 927.58$ \\
\hline Model group & 10 & $168.56 \pm 71.25$ & $94.37 \pm 37.51$ & $789.68 \pm 293.47$ & $3137.68 \pm 986.89$ \\
\hline Treatment group & & & & & \\
\hline TCF-GF(-) & 10 & $79.65 \pm 21.28$ & $45.13 \pm 15.21$ & $263.14 \pm 81.52$ & $2373.57 \pm 721.13$ \\
\hline TCF-GF(+) & 10 & $56.45 \pm 18.21$ & $29.21 \pm 10.12$ & $136.14 \pm 45.23$ & $4864.27 \pm 2071.41$ \\
\hline APB-GF(-) & 10 & $46.52 \pm 14.13$ & $24.32 \pm 8.17$ & $131.31 \pm 41.34$ & $4618.98 \pm 1861.12$ \\
\hline APB-GF(+) & 10 & $22.15 \pm 7.28$ & $10.15 \pm 3.25$ & $73.14 \pm 18.25$ & $7614.08 \pm 2071.41$ \\
\hline F & 4.003 & 5.184 & 3.523 & 5.216 \\
\hline P & 0.033 & 0.013 & 0.035 & 0.009 \\
\hline
\end{tabular}

\section{Figures}
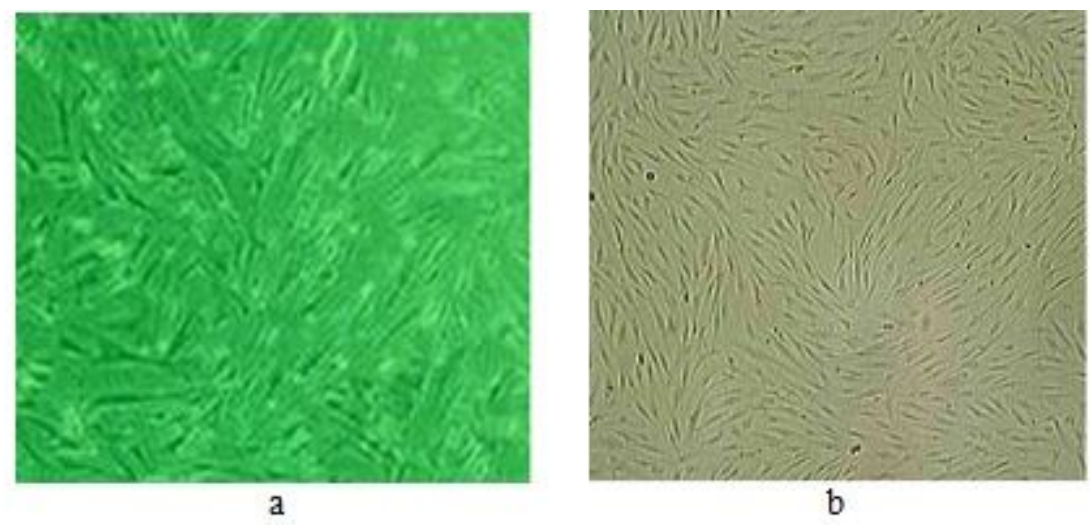

\section{Figure 1}

The morphology of BMSCs isolated from bone marrow. a)The morphology of BMSCs in Passage 1. b) The morphology of BMSCs in Passage 3. 


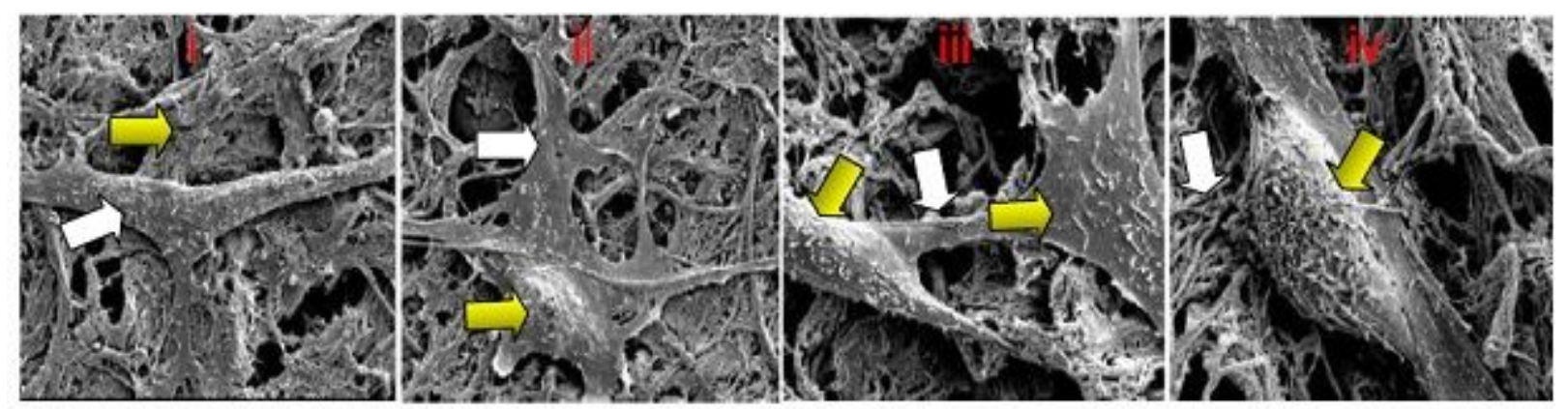

a

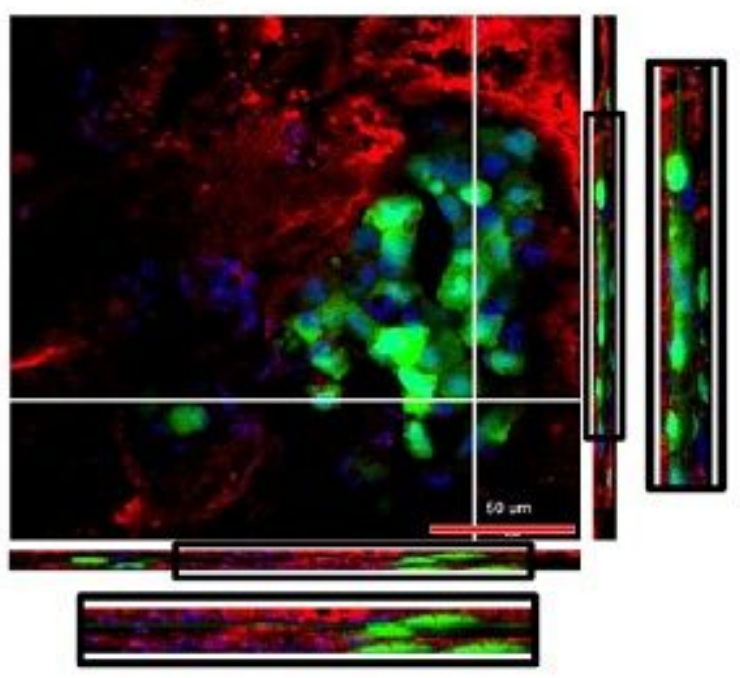

b

Figure 2

The morphology of BMSCs reseeded on APB by SEM and LSCM a)The seeded BMSCs attached on APB and the adhesion among neighboring cells by SEM(Scale bars $=1 \mu \mathrm{m})$. b)BMSCs not only attached on the surface of the bioscaffold, but also formed in cluster in the inner structure of APB indicated by LSCM (Scale bars $=1 \mu \mathrm{m})$.

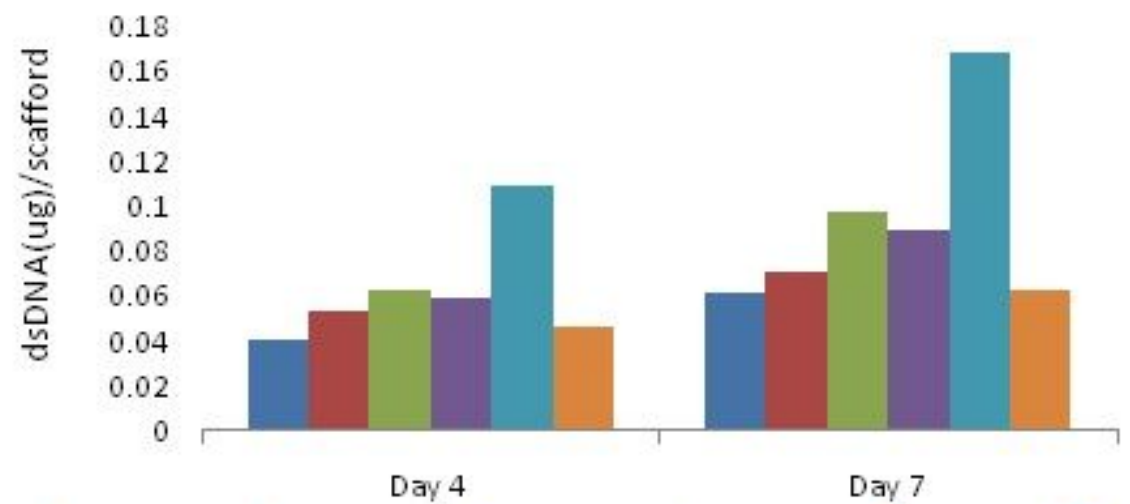

$\square 0 \mathrm{ml} / \mathrm{min} \quad 0.5 \mathrm{ml} / \mathrm{min} \quad 1 \mathrm{ml} / \mathrm{min} \square 2 \mathrm{ml} / \mathrm{min} \quad \square \mathrm{ml} / \mathrm{min} \quad \square \mathrm{ml} / \mathrm{min}$

\section{Figure 3}

DNA quantification of BMSCs in the APB on day 4 and day 7 


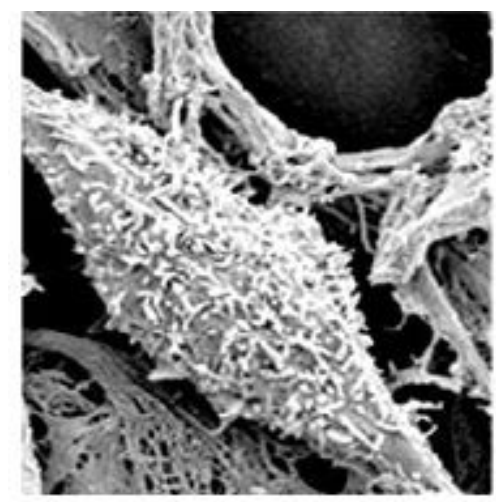

a

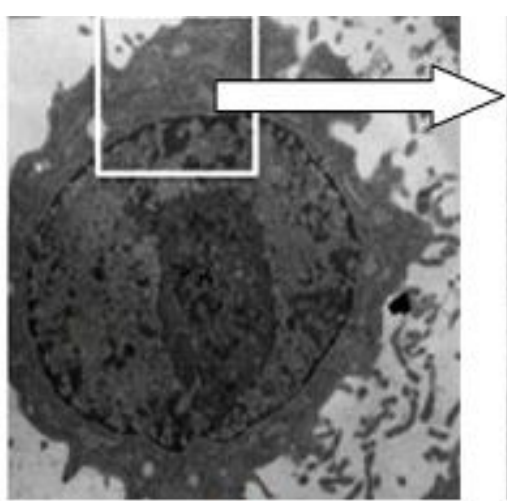

b

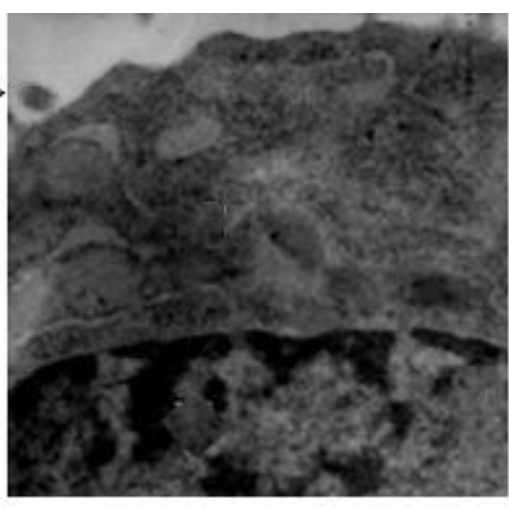

c

\section{Figure 4}

The undifferentiated BMSCs by SEM and TEM a)The undifferentiated BMSCs by SEM (Scale bars $=1 \mu \mathrm{m}$ ).

b) $($ Scale bars $=1 \mu \mathrm{m}) \mathrm{c})($ Scale bars $=300 \mathrm{~nm})$ Not any mature organelle by TEM. 


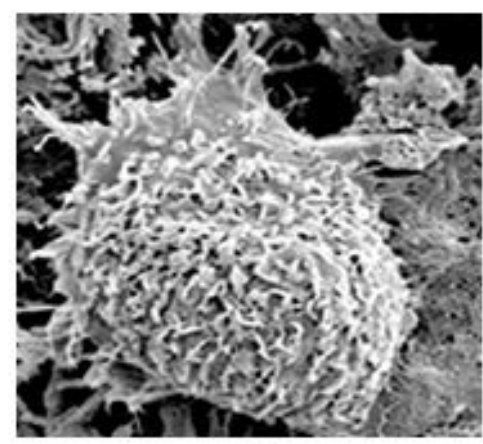

a

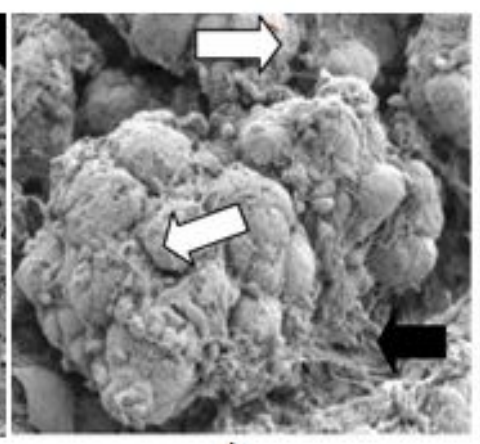

b

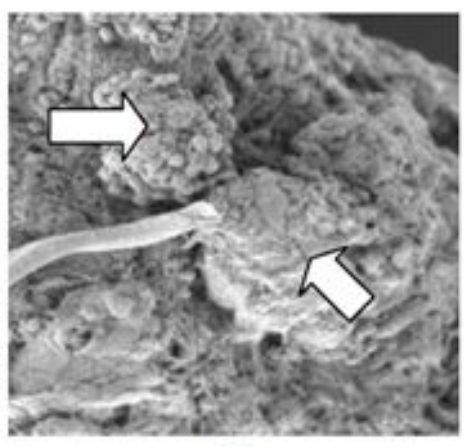

c

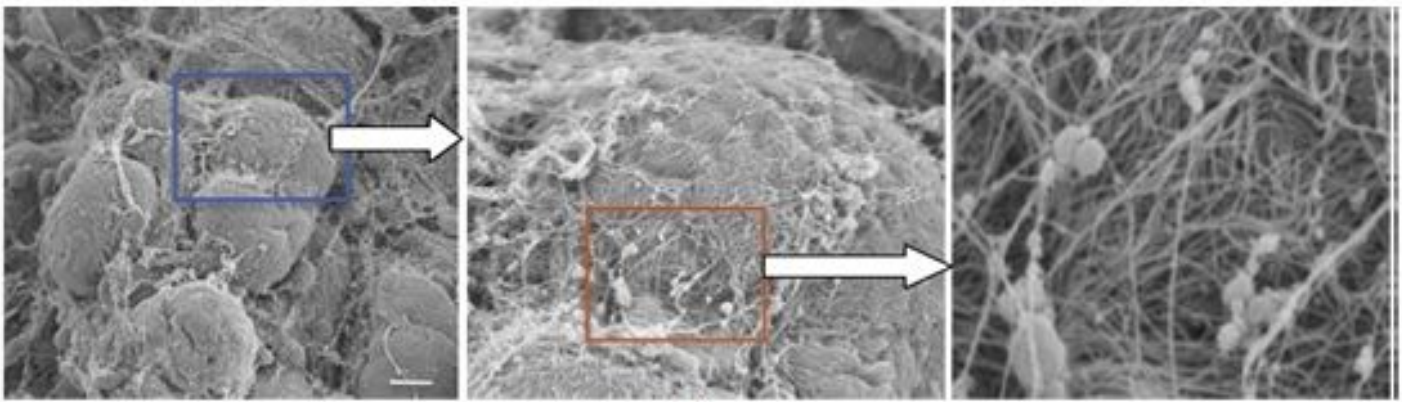

d

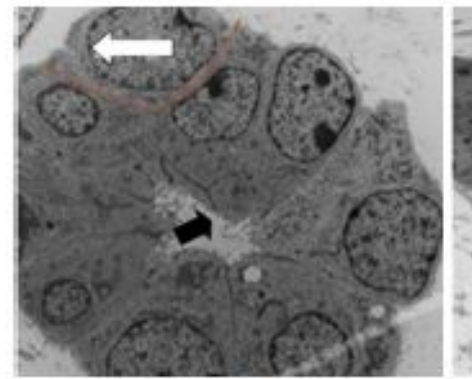

e
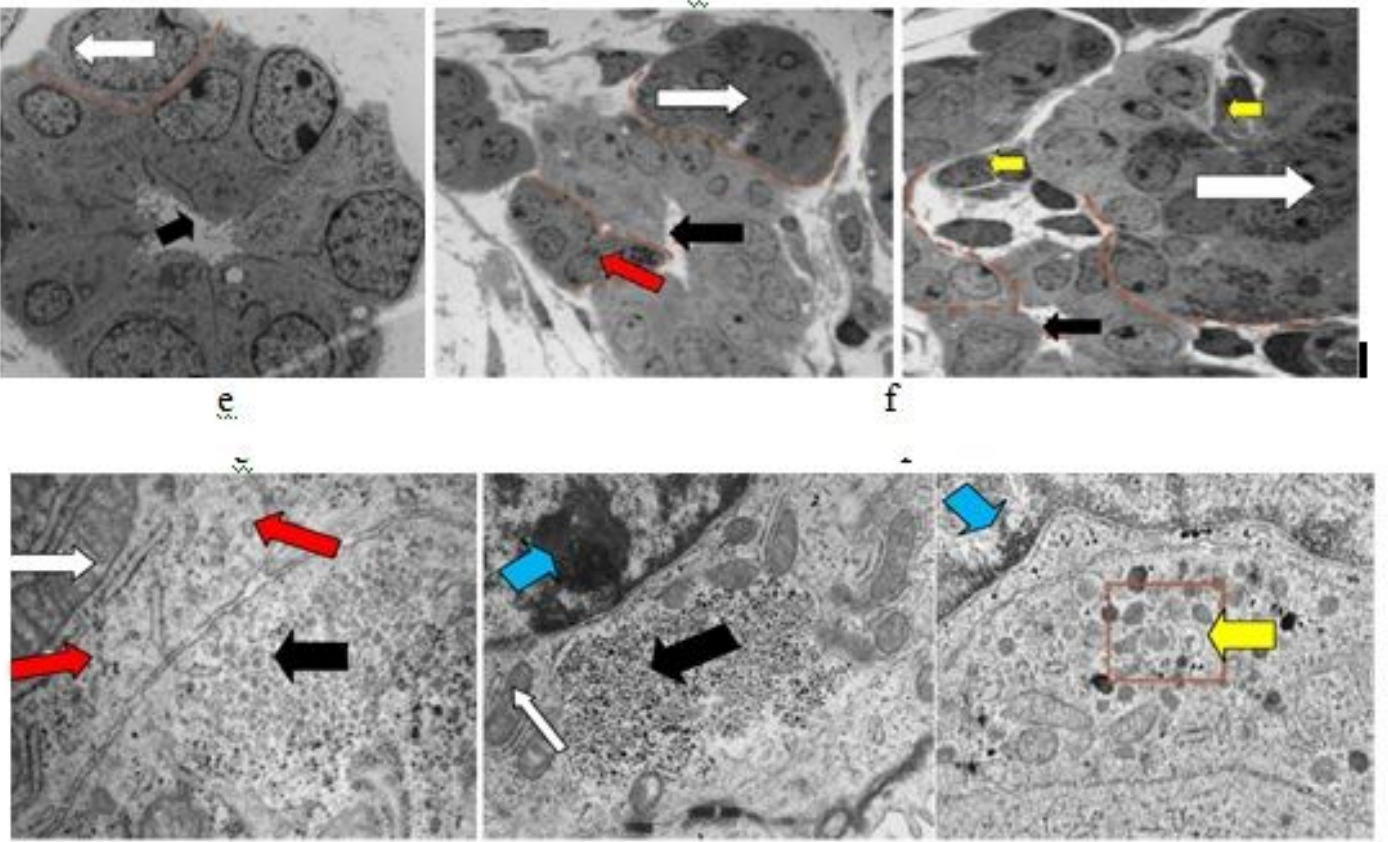

g

\section{Figure 5}

The differentiated BMSCs by SEM and TEM a) The differentiated BMSCs at the 21 st day by SEM(Scale bars $=1 \mu \mathrm{m})$. b) The increased number of epithelial-like cell clusters with complex intercalation of ECM (Scale bars $=50 \mu \mathrm{m})$. c) Differentiating endocrine and exocrine cells, and isolated small clusters(Scale bars $=50 \mu \mathrm{m})$. d) ECM accumulated around newly differentiated cells and clusters (Scale bars=10 $\mu \mathrm{m})$. e) Pancreatic-like epithelial cells organized into ductal structures by TEM(Scale bars=10 $\mu m)$. f) The 
epithelial cells in clusters separate from the ducts. Acinar cells formed amylase cell clusters. The cell clusters formed islet-like structure contained capillaries(Scale bars $=10 \mu \mathrm{m})$. g) Glycogen positivity in ductal cells, nucleus, secretory granules, mitochondria, smooth endoplasmic reticulum and rough endoplasmic reticulum in differentiated BMSCs by TEM(Scale bars $=1 \mu \mathrm{m})$.

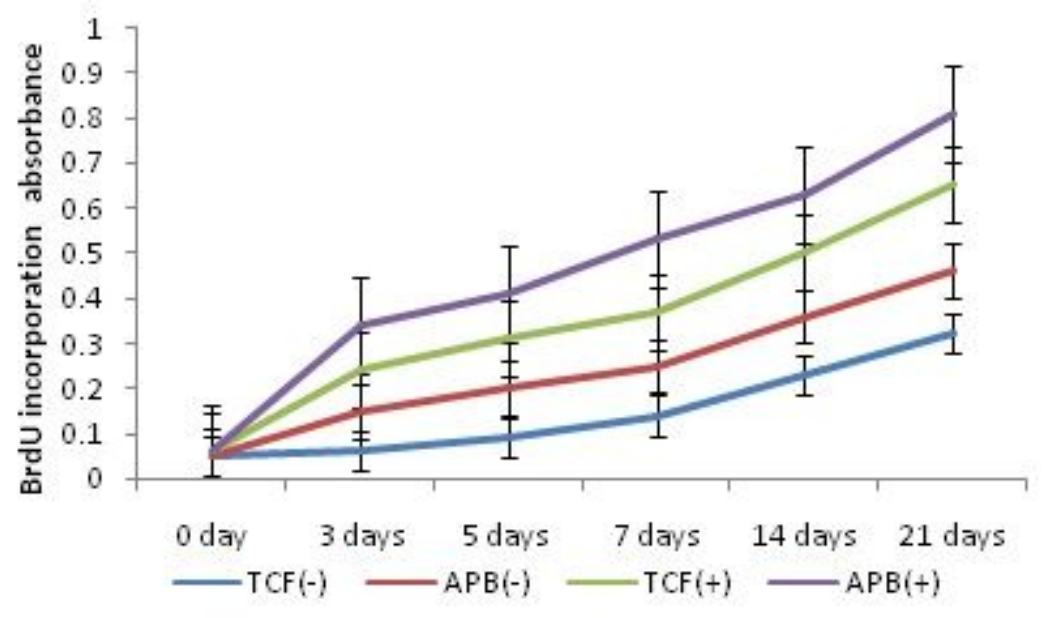

\section{Figure 6}

The proliferation of BMSCs grown on culture system 


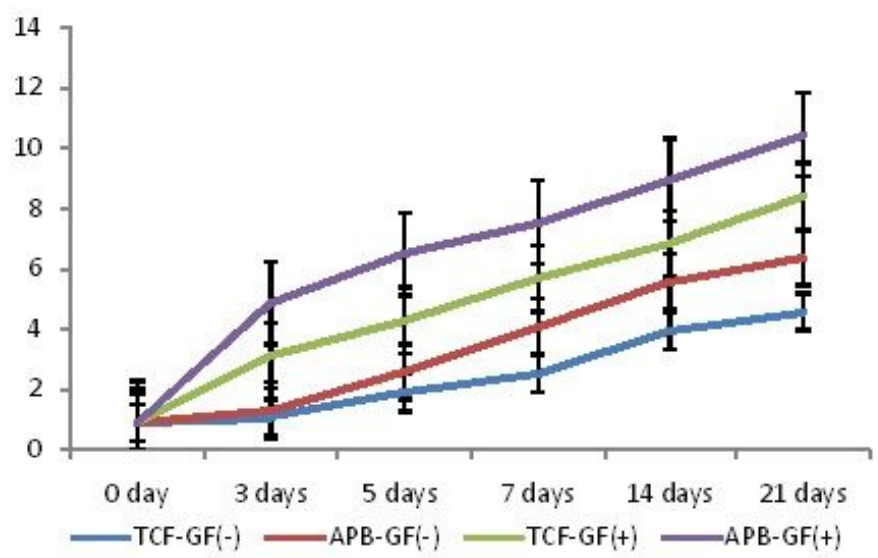

Figure $7 \mathrm{a}$

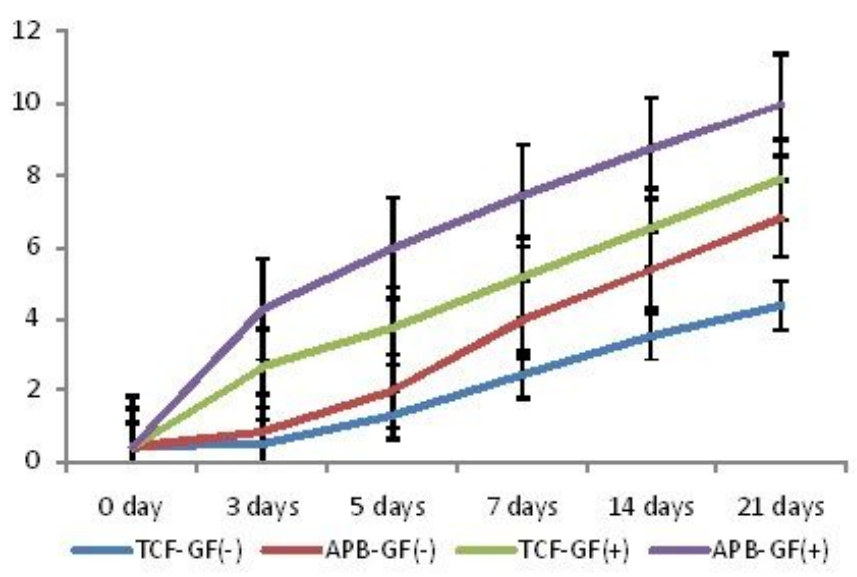

Figure $7 b$

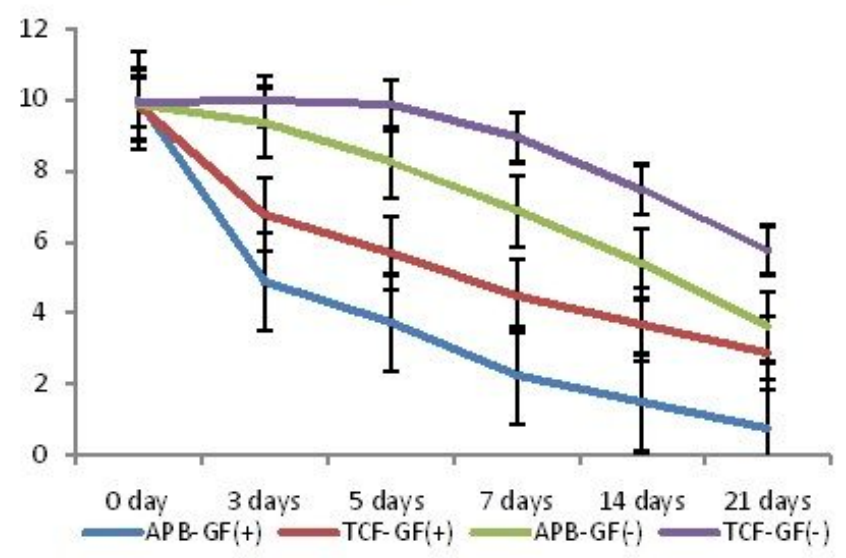

Figure $7 \mathrm{c}$

\section{Figure 7}

The expression of pancreatic and BMSCs genes a) The expression of pancreatic ancini gene PDX-1 by RT-PCR. b) The expression of pancreatic gene PTF- 1 by RT-PCR. c) The expression of BMSC gene Oct4 by RT-PCR. 

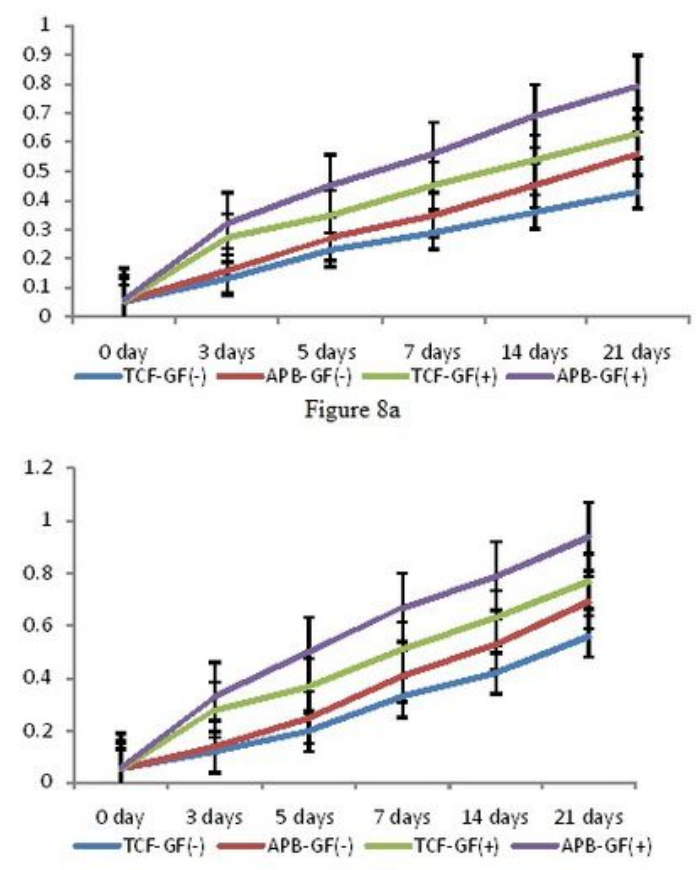

Figure $8 b$
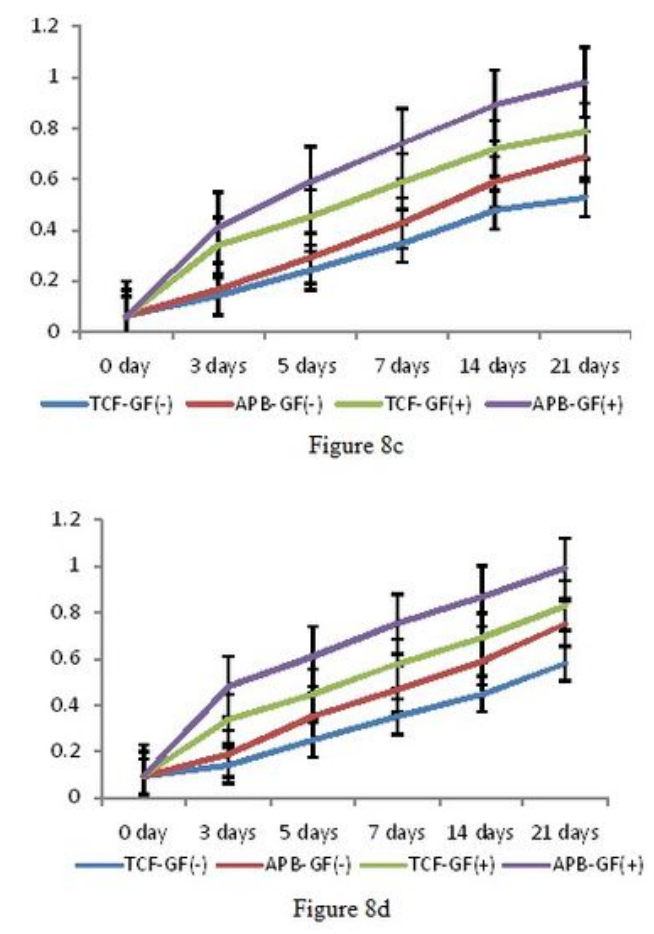

\section{Figure 8}

The expression of pancreatic cytoketatins a) The expression of pancreatic cytoketatinsa-Amy by western blot. b) The expression of pancreatic cytoketatins CK7 by western blot. c) The expression of pancreatic cytoketatins Flk-1 by western blot. d) The expression of pancreatic cytoketatins C-peptide by western blot. 


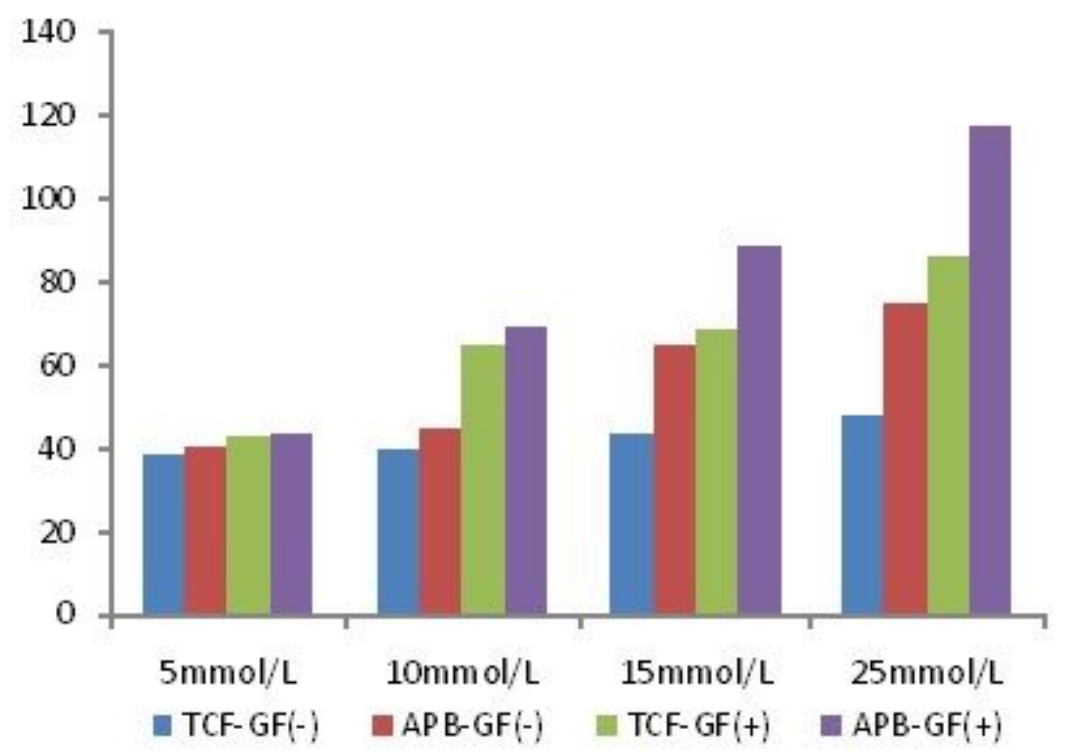

Figure 9a

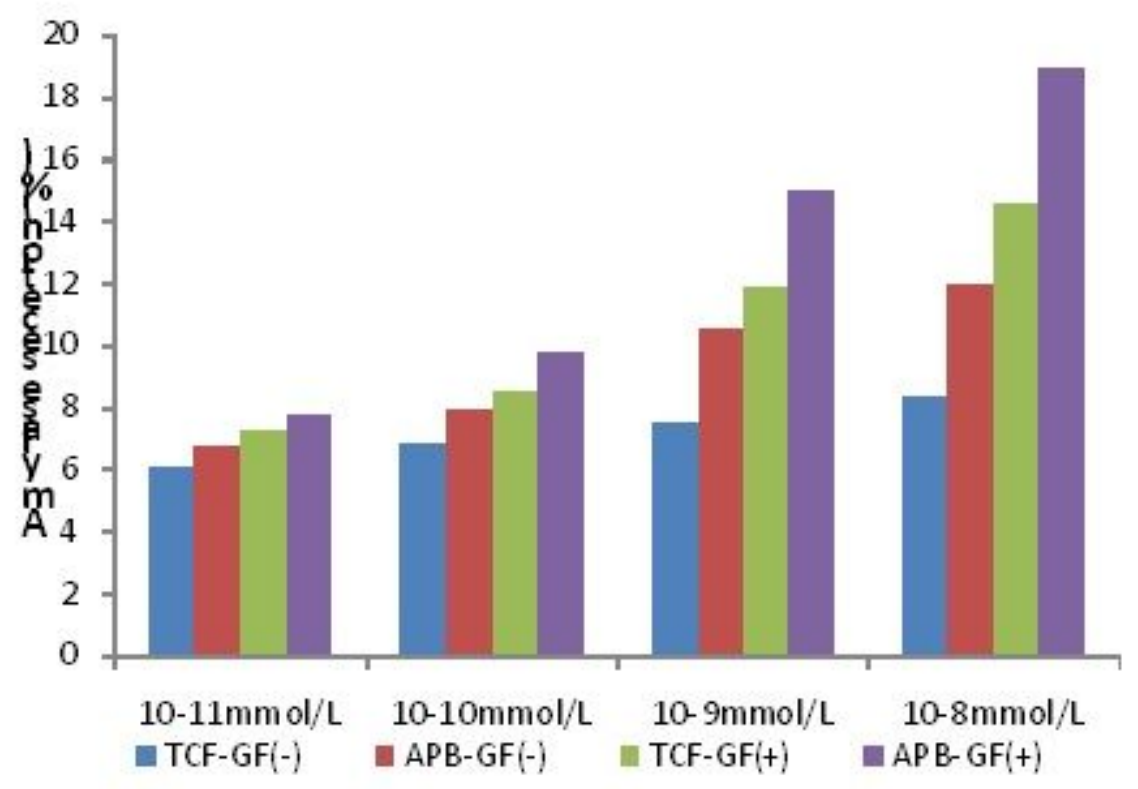

Figure $9 \mathrm{~b}$

\section{Figure 9}

The assessment of pancreatic metabolic function a) The insulin release among 4 groups at increasing glucose concentrations. b) The Amylase release among 4 groups at increasing CCK concentrations. 